\title{
Exploring the effect of cellulose nanowhiskers isolated from oil palm biomass on polylactic acid properties
}

\begin{abstract}
In this work, polylactic acid (PLA) reinforced cellulose nanowhiskers (CNW) were prepared through solution casting technique. The CNW was first isolated from oil palm empty fruit bunch microcrystalline cellulose (OPEFB-MCC) by using 64\% $\mathrm{H} 2 \mathrm{SO} 4$ and was designated as CNW-S. The optical microscopy revealed that the large particle of OPEFB-MCC has been broken down by the hydrolysis treatment. The atomic force microscopy confirmed that the CNW-S obtained is in nanoscale dimension and appeared in individual rod-like character. The produced CNW-S was then incorporated with PLA at 1,3, and 5 parts per hundred (phr) resins for the PLA-CNW-S nanocomposite production. The synthesized nanocomposites were then characterized by a mean of tensile properties and thermal stability. Interestingly to note that incorporating of $3 \mathrm{phr} / \mathrm{CNW}-\mathrm{S}$ in PLA improved the tensile strength by $61 \%$. Also, CNW-S loading showed a positive impact on the Youngô modulus of PLA. The elongation at break $(\mathrm{Eb})$ of nanocomposites, however, decreased with the addition of CNW-S. Field emission scanning electron microscopy and transmission electron microscopy revealed that the CNW-S dispersed well in PLA at lower filler loading before it started to agglomerate at higher CNW-S loading (5 phr). The DSC analysis of the nanocomposites obtained showed that $\mathrm{Tg}, \mathrm{Tcc}$ and Tm values of PLA were improved with CNW-S loading. The TGA analysis however, revealed that incopreated CNW-S in PLA effect the thermal stability (T10,T50 and Tmax) of nanocomposite, where it decrease linearly with CNW-S loading.
\end{abstract}

Keyword: Cellulose nanowhiskers; Microcrystalline cellulose; Polylactic acid; Tensile properties; Thermal analysis 